\title{
Flow Cytometric Measurement of Reactive Oxygen Species to Assess the Effects of Preconditioning Total Body Irradiation on NOG Mice
}

\author{
Maria Kavianpour ${ }^{1}$, Kobra Moradzadeh ${ }^{2}$, Samad Muhammadnejad ${ }^{2}$, Zahra \\ Jabbarpour ${ }^{2}$, Amir Arsalan Khorsand ${ }^{2}$, Sajjad Aghayan ${ }^{2}$, Mohammad Vasei ${ }^{3}$, \\ Javad Verdi ${ }^{1 *}$
}

\begin{abstract}
Background: Preclinical development of new drugs for cancer immunotherapy requires preconditioning total body irradiation (TBI) of mice to be humanized via hematopoietic stem cell transplantation. To assess the effect of preconditioning TBI, we detected the reactive oxygen species (ROS), Annexin V, propidium iodide (PI) level in bone marrow samples by flow cytometer. Methods: We divided all NOG mice between irradiated $(n=20)$ and control groups $(\mathrm{n}=10)$ for two time points. Irradiated mice were exposed to $3.5 \mathrm{~Gy}$ of radiation. After sacrificing BM samples were collected, the flow cytometric percentage of ROS, Annexin V, and PI markers were investigated on days 2 and 14 after exposure. Results: At the first time point, the level of ROS was higher in the irradiated group than in the control group, and this difference was statistically significant $(\mathrm{P}<0.05)$. Also, at the second time point, the mean differences of all markers in the irradiated group were significantly compared to the control group $(\mathrm{P}<0.05)$. Conclusion: Thus, in NOG mice, the measurement of ROS level is helpful to the assessment of preconditioning TBI.
\end{abstract}

Keywords: Irradiation- NOG Mice- bone marrow- apoptosis- reactive oxygen species- cancer immunotherapy

Asian Pac J Cancer Prev, 23 (2), 383-388

\section{Introduction}

The US Food and Drug Administration approved the immune checkpoint inhibitor (ICI) as a game-changing cancer treatment (Xu et al., 2021). ICI immunotherapy has been used to treat various cancer types such as melanoma, carcinoma, and lymphoma in the clinical setting, showing great potential for clinical application (Ferreiraet al., 2021). ICI immunotherapy research can lead to improved immunotherapy methods. Drugs that block checkpoints work by releasing $\mathrm{T}$ cells to recognize and kill tumors (Pisibonet al., 2021). Research is being conducted on many checkpoint factors, including Programmed cell death protein 1 (PD-1), cytotoxic T-lymphocyte-associated protein 4 (CTLA-4), lymphocyte activation gene 3 (LAG-3), T-cell immunoglobulin and mucin domain-3 (TIM-3), and T cell immunoreceptor with Ig and ITIM domains (TIGIT) (Deshpande et al., 2020).

These drugs are developed in preclinical trials using immunodeficiency mouse models that have been humanized with hematopoietic human stem cell (HSC) transplantation (Bagchi et al., 2021). Using mice with human immune systems and human tumors can provide useful preclinical data on cancer immunotherapy. For better engraftment of HSC in mice, they are preconditioned with total body irradiation (TBI) before HSC transplantation (Yao et al., 2019). A humanized mice for cancer immunotherapy research contains three components: human immune cells, human tumor cells, and immunodeficient host mice (De La Rochere et al., 2018). Based on current knowledge, there is no biomarker to indicate irradiation-induced damage can be justified in all mice, and it is suitable for engraftment of HSC.

As a valuable tool, flow cytometry can be used to analyze necrosis and apoptosis quickly. Using fluorescently-tagged annexin V, apoptosis can be detected by measuring the release of phosphatidylserine from the plasma membrane that Annexin $\mathrm{V}$ attaches to it, and the DNA-binding dye propidium iodide (PI), necrosis can be detected (Andrade et al., 2010; Moumariset al., 2015). Irradiation leads to oxygen-derived free radicals called reactive oxygen species (ROS) (Griendling et al., 2016). ROS trigger oxidative stress, apoptotic, and inflammatory pathways by interacting with cellular macromolecules 
such as DNA, proteins, and lipids (Schieber and Chandel, 2014). Flow cytometry can be used to assess the levels of intracellular ROS, apoptosis, and necrosis. Since mice such as NOG are used in ICI immunotherapy research and because a modification has occurred in the immune system of these mice, so far, no study has been performed to measure biomarkers for preconditioning TBI. This study aimed to comparatively evaluate the flow cytometer's effectiveness in detecting the ROS and Annexin V/PI level in BM samples following irradiation exposure in NOG mice strain.

\section{Materials and Methods}

\section{Animal experiments}

A total of 30 females (11-12 weeks old) NOD.CgPrkdcscidIl2rgtm1Sug/ShiJic (NOG) mice with similar body weights $(22.15 \pm 0.07)$ were obtained from the Digestive Disease Research Institute of Tehran University of Medical Sciences animal facility. The mice were housed in a positive, individually ventilated caging (IVC) system under controlled temperature and humidity, 12h light/12h dark cycles, and fed a sterilized pellet diet and water ad libitum. We divided the animals into two groups; Group 1 $(\mathrm{n}=20)$ was exposure total body irradiation at a sub-lethal dose of (3.5 Gy, 2.5 Gy/min, Elekta Precise accelerators), Group $2(\mathrm{n}=10)$ was used as a control group. Bone marrow samples were collected on days 2 and 14 . To anesthetize animals, we used Ketamine $(100 \mathrm{mg} / \mathrm{kg})$ and Xylazine $(10 \mathrm{mg} / \mathrm{kg})$ mixture in $1 \mathrm{x}$ PBS, then by cervical dislocation were euthanized.

Procedures were carried out according to a protocol that the Ethical Committee approved of Tehran University of Medical Sciences (IR.TUMS.MEDICINE. REC.1399.227).

\section{Flow cytometry Analysis}

High-quality staining is achieved by combining Annexin V with PI, a DNA intercalating counterstain often used to distinguish between apoptotic and necrotic cells in a population. The Annexin V-PI staining procedure (BD, Cat. No. 556547) (Riegeret al., 2011). The protocol consists of (i) washing cells once with cold 1x PBS, (ii) suspending one million cells in $1 \mathrm{x}$ binding buffer (100 $\mu \mathrm{l})$, (iii) adding Annexin V $(5 \mu \mathrm{l})$ and PI $(5 \mu \mathrm{l})$, and (iv) completing the volume to $500 \mu \mathrm{l}$ with $1 \mathrm{x}$ binding buffer. The cells were stored in the dark until they were assessed, and then flow cytometry was used to evaluate them within one hour. Untreated cells were gated at the FSC-SSC (forward scatter-side scatter) plot and percentages felt into four zones were computed.

The mean fluorescence intensity (MFI) of dye $2^{\prime}, 7^{\prime}$-dichlorofluorescein diacetate (DCFH-DA) was measured using a flow cytometer to evaluate the productions of ROS in BM samples. DCFH-DA is a stable, non-fluorescent, cell-permeable compound, which, penetrating the cell, is converted to DCFH by intracellular esterase, is trapped within the cell, and is stable for a few hours. On oxidation, ROS de-esterified product is transformed to the highly fluorescent $2^{\prime}, 7^{\prime}$ dichlorofluorescein (DCF), which emits green fluorescence when excited at $488 \mathrm{~nm}$, proportionate to the intracellular level of ROS. DCFH-DA $(10 \mu \mathrm{M})$ was added to the BM-mononuclear cells and incubated at $37^{\circ} \mathrm{C}$ for 30 $\mathrm{min}$ in the dark. Cells were then washed, resuspended in PBS, and detected by flow cytometry. Data were analyzed using the FlowJo 7.6.1 software (Ashland, OR, USA).

\section{Histopathologic Analysis}

At 2, 14 days after irradiation, the mice were sacrificed, and BM samples were harvested, fixed for $48 \mathrm{hr}$ in $10 \%$ neutral buffered formalin, embedded in paraffin, sectioned at five $\mu \mathrm{m}$, and stained with hematoxylin and eosin (H\&E). Histological evaluation of sections was performed as the gold standard to confirm irradiation-induced injury in the BM sample.

\section{Statistical analysis}

The implantation rates were compared to determine the effect of exposure to irradiation on BM injury. The Mann-Whitney U test was used to compare differences between groups. Differences were considered significant at $\mathrm{P}<0.05$. The results are reported as the median, minimum, and maximum range. Data analysis was performed with GraphPad Prism 9 for Windows (GraphPad Software, Inc; CA).

\section{Results}

After exposure to 3.5 Gy of irradiation, irradiated mice $(n=20)$ and the control group $(n=10)$ were sacrificed at two different time points ( 2 and 14 days after exposure). Apoptosis, necrosis, and ROS in BM samples were measured by flow cytometry. In time point 1, data showed mean differences in the intracellular amount of ROS was significant compared to the control group $(\mathrm{P}<0.05)$ (Figure 1$)$. However, there was no significant difference between the irradiated group and control for early and late apoptosis or necrosis at this time point. Also, the results demonstrated that in time point 2, apoptosis, necrosis, and ROS significantly increased in the irradiated group compared to control $(\mathrm{P}<0.05)$ (Figure 2 ). To confirm the extent of damage after irradiation, histological examination of BM samples was performed with H\&E staining and checked by pathologist (Figure 3).

\section{Discussion}

Today, cancer immunotherapy for ICI is one of the hot topics in the scientific literature, and their drug development platform is based on humanized mice (Chen and Mellman, 2017). To create humanized mice, immunodeficiency mice such as NSG, SCID, NOD, and NOG are used whose immune system is genetically engineered and $\mathrm{T}, \mathrm{B}$, and NK cell-deficient also macrophage and dendritic cell function is reduced (De La Rochere et al., 2018). After exposure to radiation and induction of preconditioning TBI, HSC is transplanted to induced immune systems similar to humans. The tumor is injected or transplanted into mice, then ICI-based drugs are intervened to evaluate the tumor's able effect (Strowig et al., 2011). However, the fact is that no test was performed to evaluate the effect 
A

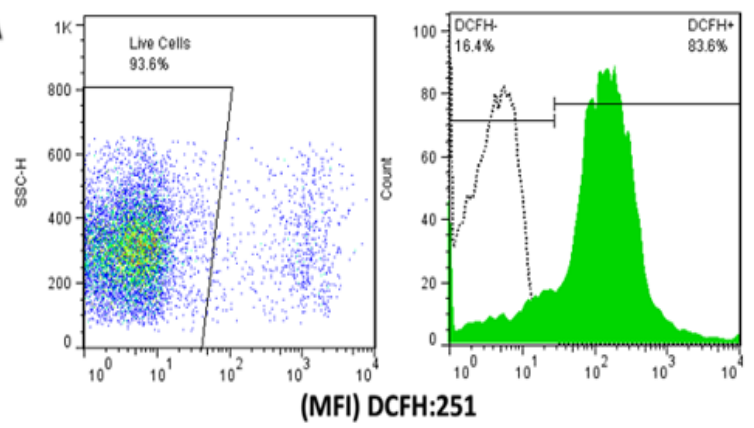

C

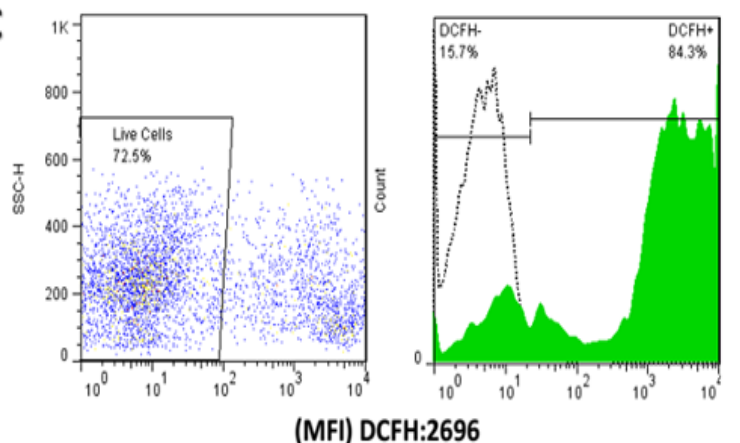

B

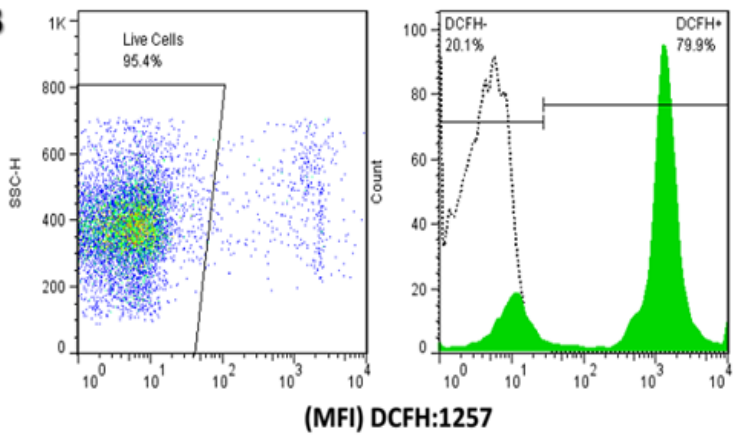

ROS

D

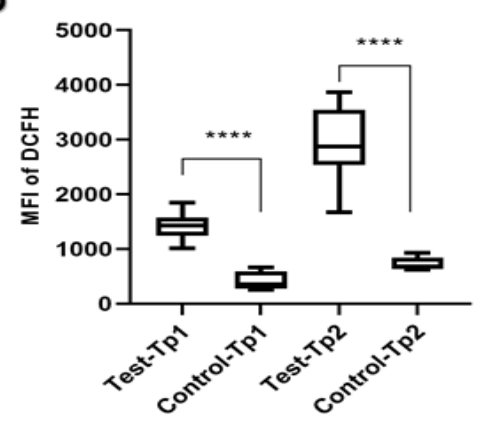

Time points

Figure 1. Flow Cytometry with DCFH-DA Staining for Measuring ROS Production in BM Samples after Radiation Exposure (Diagram showing one of the study repetitions). Flow cytometry plots were shown in A, B, and C for Control, Timepoints 1, and 2, respectively. D Graph presents Mann-Whitney U analysis of MFI of DCFH in all BM sample of groups. (The data presented are means \pm SEM. ns: not significant, * p-value $<0.05$, ** p-value $<0.01$ and $* * *$ p-value $<0.001)$.

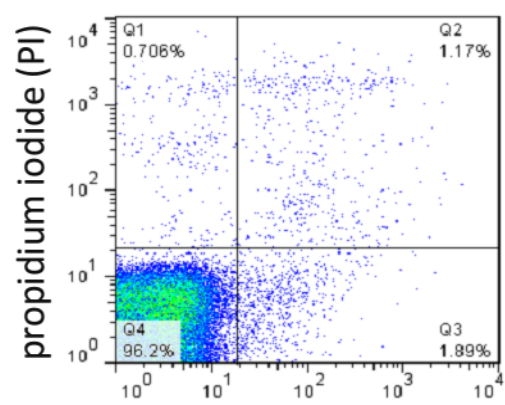

A Annexin V

D

Darly Apoptosis

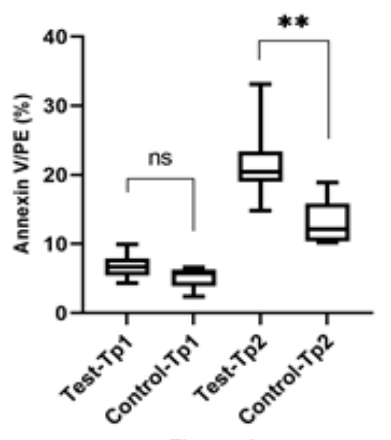

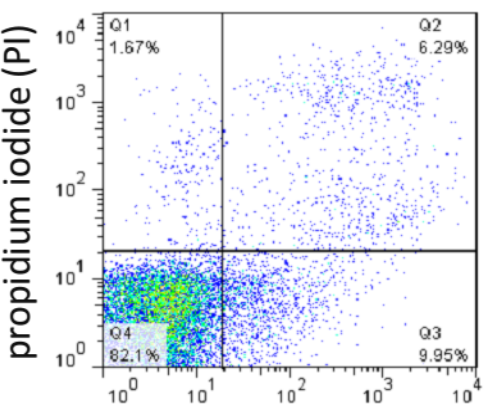

B Annexin V

Late Apoptosis

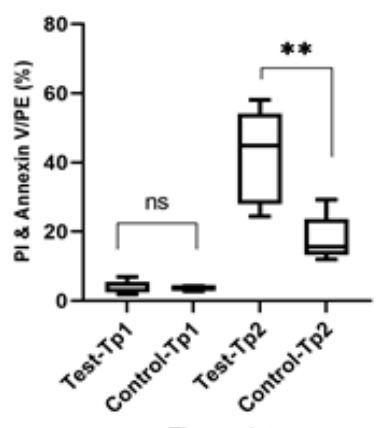

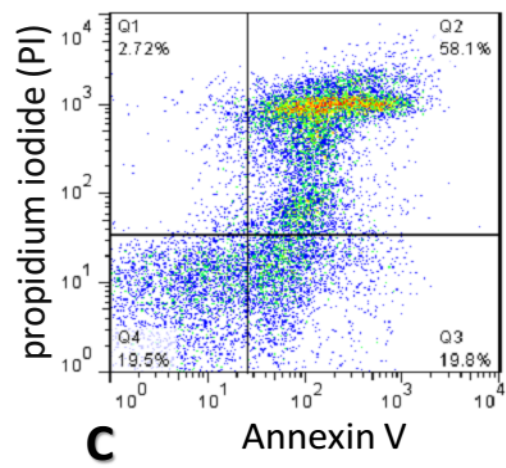

Necrosis

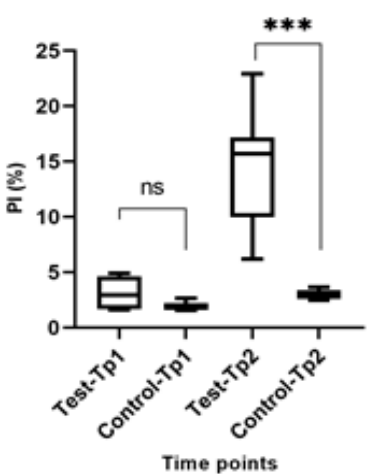

Figure 2. Flow Cytometry Analysis of Annexin-V and Propidium Iodide (PI) Staining of Apoptosis and Necrosis (Diagram showing one of the repetitions of the study). A) Control sample, B) Timepoint 1 (day 2), C) Timepoint 2 (day 14); D) Graphs showing the percentage of apoptotic cells (annexin V positive for early apoptosis, PI and annexin $\mathrm{V}$ double-positive cells for late apoptosis, and PI-positive for the percentage of necrosis) (The data presented are means \pm SEM. ns: not significant, ${ }^{*}$ p-value $<0.05,{ }^{* *}$ p-value $<0.01$ and $* * *$ p-value $\left.<0.001\right)$. 


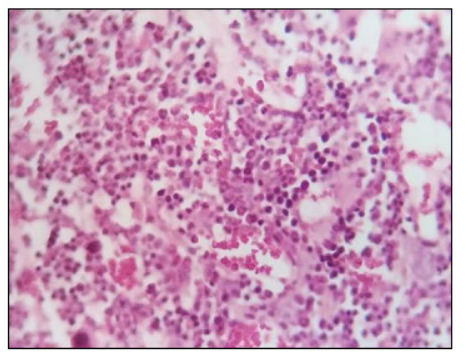

Control

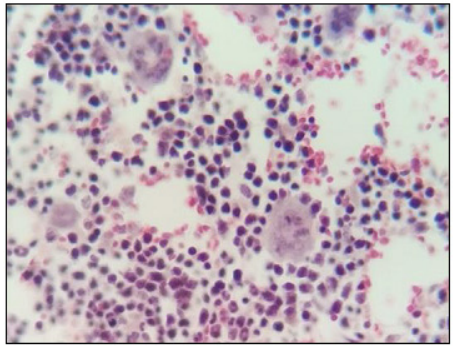

Day 2

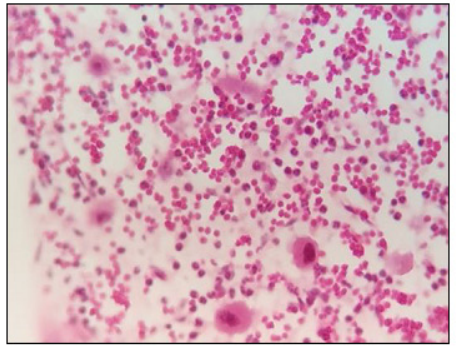

Day 14

Figure 3. The Pathological Findings of the NOG Mice BM were Shown after being Exposed to 3.5 Gy Total Body Irradiation. Radiation-induced cellularity depletion of BM tissue occurred after irradiation and was more reduced on day $14(50 \%)$ than on day $2(30 \%)$. BM sections were stained with hematoxylin and eosin (H\&E) (Scale bar: $400 \mu \mathrm{m})$.

of the TBI in this mouse strain and to show the amount of damage caused by irradiation. Therefore, it is felt that a biomarker is needed to show us whether the amount of damage is desirable for HCS engraftment and whether this damage has been induced in all mice (Boieri et al., 2016). To find an early detection marker, we determined ROS, apoptosis, and necrosis by flow cytometry. Our goal was to identify a suitable flow cytometry marker among them within a few days of irradiation. At the first time point, the mean difference ROS level was higher than other markers like Annexin V and PI, that this marker increased before apoptosis or necrosis induction.

In line with our study, Andrade and his colleagues studied the effects of sublethal irradiation on engraftment after murine BM transplantation. This study using the flow cytometry method on day 3 showed no increase in the number of donor cells in irradiated bone marrow, confirming that sublethal irradiation does not enhance bone marrow chimerism early after transplantation. Nevertheless, irradiation significantly increased late donor chimerism. Finally, they showed that the combination of TBI and intravenous administration of donor cells provided the highest levels of long-term donor chimerism in the marrow space, and the use of sublethal irradiation enhances homing (Andrade et al., 2011). So, sub-lethal irradiation as a preconditioning method could be helpful. The only study so far similar to ours to investigate preconditioning with irradiation reported by Andersen et al. on NOG mice. In this study, they performed the effect of Cesium-137 versus X-ray irradiation on humanized mice to examine the humming of human cells in mouse bone marrow. They concluded that no significant differences between the two sources in the low-dose spectrum were investigated. Both X-Ray and Cs-137 sources could sufficiently ablate the mouse bone marrow to allow human stem cell engraftment. They recommend that all researchers consider technical specifications and dose-finding studies (Andersen et al., 2020). However, no test is performed to optimize the dose of radiation and its effects.

According to Lorimore et al.'s study, activated macrophages and inflammatory-type reactions occurred in the hemopoietic system after exposure to ionizing irradiation. Based on their results, increased Nitric oxide synthase (NOS) enzyme activity and neutrophil infiltration in the tissue, activation of macrophages provide a mechanism for damage production through a "bystander" effect, which may contribute to irradiation-induced genomic instability and leukemogenesis (Lorimore et al., 2001). Phosphorylated histone H2AX, Caspase 3, and TUNEL were measured by immunocytochemistry 24 hours after irradiation in a study by Burr et al., (2010) as a result, their findings showed that irradiation exposure resulted in the formation of a damaging BM microenvironment 24 hours after irradiation and that macrophages could cause secondary damage following the initial irradiation-induced injury. This delayed bystander effect highlighted the relevance of tissue responses. Their study results were inconsistent with ours because macrophages play a critical role in this mechanism, whereas we used the immunodeficient NOG mice that have reduced macrophage function. Due to this mechanism, increased apoptosis and necrosis might be delayed, and in NOG mice, an early marker is needed.

In another study, irradiation at ranges of 2 to $20 \mathrm{~Gy}$ was used that did not cause direct cellular apoptosis or necrosis but induced mitochondrial damage in cells; thus, an easier and faster method is needed (Zhou et al., 2021). In another study, the level of apoptosis in two mice strains (C57 and CBA) in different irradiation doses was assessed. The results demonstrated that apoptosis levels differed between the strains with CBA that apoptosis levels were higher at $24 \mathrm{~h}$ than C57, but C57 showed a higher level of apoptosis at the delayed time point (Irons et al., 2012). However, in our study, NOG mice had a higher level of apoptosis on day 14 than two days after irradiation; this may be due to differences in the strains of the study mice from other studies. On the other hand, these mice have low irradiation tolerance, and basically, doses higher than 4 Gy cannot be used for this strain of mice (Shultz et al., 2005). Mukherjee et al. investigated the relationships between apoptotic responses of cells exposed and tissue cytotoxicity in irradiated (0.25-2 Gy) murine BM. Up to $24 \mathrm{~h}$ post-irradiation, BM cellularity showed reductions, but in vivo apoptosis measurements were not detected. There was ongoing cell death up to $24 \mathrm{~h}$ post-irradiation, while the level of apoptosis is not elevated and cytokines are produced in response to the initial tumor protein 53 (p53)-induced apoptosis. In the presence of low levels of measured apoptosis, intramedullary cell death and 
apoptotic processes associated with pro-inflammatory mechanisms contribute to additional ongoing cell death (Mukherjee et al., 2013). A lack of immune cells (B-cells and T-cells, macrophages, and natural killer cells (NK)) and pro-inflammatory mechanisms in NOG mice has caused BM damage to be delayed (Liouet al., 2011; Shultz et al., 2000).

Our study had strengths and weaknesses; we assessed the early detection marker for preconditioning success. We did not assess immunocytochemistry tests such as TUNEL or apoptotic and necrotic factors following budget constraints.

In cancer immunotherapy studies, humanized mouse models are a promising tool for new treatments that can play an important role in treating and selecting humanspecific immunomodulatory agents and even designing personalized studies. Therefore, studying these animal models is very important to improve their performance in future studies. In this study, ROS was introduced as a flow cytometry biomarker for determining the effect of preconditioning TBI on the NOG mice to humanize them. In future preclinical cancer immunotherapy studies, the level of preconditioning success could be evaluated using $\mathrm{BM}$ aspiration and intracellular ROS measurements.

\author{
Abbreviations \\ BM: Bone Marrow \\ Gy: Gray \\ NOG: NOD/Shi-scid/IL-2R $\gamma$ null \\ ROS: reactive oxygen species
}

\section{Author Contribution Statement}

MK was the major contributor in literature search writing the manuscript, and spelling and grammar check. $\mathrm{KM}, \mathrm{ZJ}$, and SA managed to collect tissues. AAKH, SM, and MV conducted experiments. JV The main supervisor of the search and corresponding author. All authors read and approved the final manuscript.

\section{Acknowledgments}

\section{Ethics approval}

This study was conducted under a protocol approved by the Ethical Committee of Tehran University of Medical Sciences (IR.TUMS.MEDICINE.REC.1399.227).

\section{Consent for publication}

All of the authors declare that they have all participated in the design, execution, and analysis of the paper, and that they have approved the final version.

Availability of data and material not applicable.

\section{Funding statement}

This study was funded by a grant from the Tehran University of Medical Sciences (grant number: 98-0287-43338). This paper is issued from the thesis of Maria Kavianpour.

\section{Conflicts of interest}

The authors have no conflicts of interest to declare that are relevant to the content of this article.

\section{References}

Andersen AHF, Nielsen SSF, Olesen R, et al (2020). Comparable human reconstitution following Cesium-137 versus X-ray irradiation preconditioning in immunodeficient NOG mice. PLoS One, 15, e0241375.

Andrade J, Ge S, Symbatyan G, et al (2011). Effects of sublethal irradiation on patterns of engraftment after murine bone marrow transplantation. Biol Blood Marrow Transplant, 17, 608-19.

Andrade R, Crisol L, Prado R, et al (2010). Plasma membrane and nuclear envelope integrity during the blebbing stage of apoptosis: a time-lapse study. Biol Cell, 102, 25-35.

Bagchi S, Yuan R, Engleman EG (2021). Immune checkpoint inhibitors for the treatment of cancer: Clinical Impact and Mechanisms of Response and Resistance. Annu Rev Pathol, 16, 223-49.

Boieri M, Shah P, Dressel R, et al (2016). The Role of Animal Models in the Study of Hematopoietic Stem Cell Transplantation and GvHD: A Historical Overview. Front Immunol, 7, 333.

Burr KL, Robinson JI, Rastogi S, et al (2010). Radiation-induced delayed bystander-type effects mediated by hemopoietic cells. Radiat Res, 173, 760-8.

Chen DS, Mellman IJN (2017). Elements of cancer immunity and the cancer-immune set point. Nature, 541, 321-30.

De La Rochere P, Guil-Luna S, et al (2018). Humanized mice for the study of immuno-oncology. Trends Immunol, 39, 748-63.

Deshpande RP, Sharma S, Watabe K (2020). The confounders of cancer immunotherapy: Roles of Lifestyle, Metabolic Disorders and Sociological Factors. Cancers, 12, 2983.

Ferreira M, Secher T, Heuze-Vourc HN, et al (2021). Immune checkpoint and anti-angiogenic antibodies for the treatment of non-small cell lung cancer in the European Union and United States. Pharmaceutics, 13.

Griendling KK, Touyz RM, Zweier JL, et al (2016). Measurement of reactive oxygen species, reactive nitrogen species, and redox-dependent signaling in the cardiovascular system: A Scientific Statement From the American Heart Association. Circulation Res, 119, e39-e75.

Irons SL, Serra V, Bowler D, et al (2012). The effect of genetic background and dose on non-targeted effects of radiation. Int J Radiat Biol, 88, 735-42.

Liou J-T, Liu F-C, Mao C-C, et al (2011). Inflammation confers dual effects on nociceptive processing in chronic neuropathic pain model. Anesthesiology, 114, 660-72.

Lorimore SA, Coates PJ, Scobie GE, et al (2001). Inflammatorytype responses after exposure to ionizing radiation in vivo: a mechanism for radiation-induced bystander effects? Oncogene, 20, 7085-95.

Moumaris M, Rajoelya B, Abuafa NJOBSJ (2015). Fluorescein isothiocyanate-dextran can track apoptosis and necrosis induced by heat shock of peripheral blood mononuclear cells and HeLa cells. Open Biol Sci J, 1.

Mukherjee D, Coates PJ, Rastogi S, et al (2013). Radiationinduced bone marrow apoptosis, inflammatory bystandertype signaling and tissue cytotoxicity. Int $J$ Radiat Biol, 89, 139-46.

Pisibon C, Ouertani A, Bertolotto C, et al (2021). Immune checkpoints in cancers: From Signaling to the Clinic. Cancers, 13, 4573.

Rieger AM, Nelson KL, Konowalchuk JD, et al (2011). Modified annexin $\mathrm{V} /$ propidium iodide apoptosis assay for accurate assessment of cell death. J Vis Exp, 2011, 2597.

Schieber M, Chandel NS (2014). ROS function in redox 
signaling and oxidative stress. Curr Biol, 24, 453-62.

Shultz LD, Lang PA, Christianson SW, et al (2000). NOD/ LtSz-Rag1null mice: an immunodeficient and radioresistant model for engraftment of human hematolymphoid cells, HIV infection, and adoptive transfer of NOD mouse diabetogenic T cells. J Immunol, 164, 2496-507.

Shultz LD, Lyons BL, Burzenski LM, et al (2005). Human lymphoid and myeloid cell development in NOD/LtSzscid IL2R $\gamma$ null mice engrafted with mobilized human hemopoietic stem cells. J Immunol, 174, 6477-89.

Strowig T, Rongvaux A, Rathinam C, et al (2011). Transgenic expression of human signal regulatory protein alpha in Rag2-l- $\gamma \mathrm{c}-1-$ mice improves engraftment of human hematopoietic cells in humanized mice. Proc Natl Acad Sci US A, 108, 13218-23.

Xu S, Sharma UC, Tuttle C, et al (2021). Immune checkpoint inhibitors: Cardiotoxicity in Pre-clinical Models and Clinical Studies. Front Cardiovasc Med, 8, 619650.

Yao LC, Aryee KE, Cheng M, et al (2019). Creation of PDXBearing Humanized Mice to Study Immuno-oncology. Methods Mol Biol, 1953, 241-52.

Zhou Z, Zhao J, Hu K, et al (2021). Single high-dose radiation enhances dendritic cell homing and $\mathrm{T}$ cell priming by promoting reactive oxygen species-induced cytoskeletal reorganization. Int J Radiat Oncol Biol Phys, 109, 95-108.

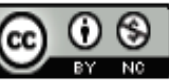

This work is licensed under a Creative Commons AttributionNon Commercial 4.0 International License. 